\title{
Hierarchical Adaptive Means (HAM) Clustering for Hardware-Efficient, Unsupervised and Real-time Spike Sorting
}

\author{
Sivylla E. Paraskevopoulou ${ }^{a, b}$, Di Wu ${ }^{a}$, \\ Amir Eftekhar ${ }^{a, b}$, and Timothy G. Constandinou ${ }^{a, b}$ \\ ${ }^{a}$ Department of Electrical and Electronic Engineering, Imperial College London, SW7 2BT, UK \\ ${ }^{b}$ Centre for Bio-Inspired Technology, Institute of Biomedical Engineering, Imperial College London, SW7 2AZ, UK \\ Email:\{s.paraskevopoulou09, a.eftekhar, t.constandinou\}@imperial.ac.uk
}

\begin{abstract}
This work presents a novel unsupervised algorithm for real-time adaptive clustering of neural spike data (spike sorting). The proposed Hierarchical Adaptive Means (HAM) clustering method combines centroid-based clustering with hierarchical cluster connectivity to classify incoming spikes using groups of clusters. It is described how the proposed method can adaptively track the incoming spike data without requiring any past history, iteration or training and autonomously determines the number of spike classes. Its performance (classification accuracy) has been tested using multiple datasets (both simulated and recorded) achieving a near-identical accuracy compared to $k$-means (using 10-iterations and provided with the number of spike classes). Also, its robustness in applying to different feature extraction methods has been demonstrated by achieving classification accuracies above $80 \%$ across multiple datasets. Last but crucially, its low complexity, that has been quantified through both memory and computation requirements makes this method hugely attractive for future hardware implementation.
\end{abstract}

Keywords: clustering, spike sorting, realtime, hardware implementable, streaming, brain machine interface

\section{Introduction}

Brain Machine Interfaces (BMIs) have attracted considerable interest in the scientific community in an effort to map neuronal activity to sensory and motor functions. With recent advances in microfabrication processes, electrodes that penetrate cortical tissue can number in the 100's, with each electrode observing the activity of up to 10 neurons (Pedreira et al., 2012). Together with modern microelectronic design, this has enabled tools capable of recording 100's of channels (Stevenson and Kording, 2011).

However, as the number of recording channels scale it becomes an increasingly challenging demand on microelectronics to transmit this information. Limits in the communication bandwidth of implantable devices, fundamentally constrained by the energy budget and the maximum allowable thermal dissipation (to avoid tissue damage) simply do not allow for such large volumes of neuronal data to be transmitted in real-time (Patil and Turner, 2008; Sarpeshkar et al., 2008)

It has therefore been the focus of many studies to find hardware implementable methods capable of reducing the data rate while maintaining accuracy in spike sorting (Barsakcioglu et al., 2013). Spike sorting being the class of methods used to isolate and identify the up to 10 neurons on each recording channel. These reduction methods can simply be in the form of data compression but several works have demonstrated hardware implementable feature extraction methods. These includes those based on derivative (Gibson et al., 2008; Paraskevopoulou et al., 2013), templates (Rizk et al., 2009), zero crossings (Kamboh and Mason, 2012) and neuronal spike shape and area (Zviagintsev et al., 2005), to name a few.

Clustering, the final step in separating spike features in the multi-dimensional feature space as to identify individual neurons, is typically achieved after transmission, either on a DSP/FPGA platform, or offline on a workstation. If however, the spike event together with the originating neuron can be locally determined, i.e. within the implant, then the data rate can be massively reduced. Until recently, the consensus was to implement the classification process after transmission because of its high complexity. For this there are a num- 
Table 1: Review of reported on-chip spike sorting implementations showing key features

\begin{tabular}{ccccccccc}
\hline Ref. & Year & $\begin{array}{c}\text { Spike } \\
\text { detection }\end{array}$ & $\begin{array}{c}\text { Feature } \\
\text { extraction }\end{array}$ & Clustering & $\begin{array}{c}\text { Number of } \\
\text { channels }\end{array}$ & $\begin{array}{c}\text { Power } \\
\left(\frac{\mu W}{\text { channel }}\right)\end{array}$ & $\begin{array}{c}\text { Tech } \\
(\mu \mathrm{m})\end{array}$ & $\begin{array}{c}\text { Area } \\
\left(\frac{\mathrm{mm}^{2}}{\text { channel }}\right)\end{array}$ \\
\hline (Karkare et al., 2011) & 2011 & NEO & DD & - & 64 & 2.03 & 0.09 & 0.06 \\
(Chen et al., 2012) & 2012 & NEO & DWT/PCA & k-means & 128 & 0.68 & 0.09 & 0.07 \\
*(Kamboh and Mason, 2012) & 2012 & DT & ZCF & Mahalanobis & - & - & - & - \\
(Karkare et al., 2013) & 2013 & Abs & PP & modified k-means & 16 & 4.68 & 0.065 & 0.07 \\
*(Saeed and Kamboh, 2013) & 2013 & NEO & ZCF & MCK classifier & - & - & - & - \\
\hline
\end{tabular}

*This paper shows the potential of hardware implementation.

ber of highly accurate software-based algorithms, such as WaveClus (Quiroga et al., 2004), Osort (Rutishauser et al., 2006) and KlustaKwik (Harris et al., 2000).

More recently however, there has been some effort to implement on-chip clustering for BMI applications (see Table 1). The still remaining challenge however is to implement a real-time, calibration-free clustering methodology of low complexity and suitable for channel scaling and showing comparable accuracy to offchip/offline methods. This work offers a potential computational methodology towards this aim.

\subsection{Feature extraction}

Feature extraction simplifies the classification process of spikes by selecting the features that best describe and separates the neuronal spikes. So for a spike consisting of $n$ sample points, the feature extraction method produces $m$ variables $(m<n)$, where $m$ is the number of features. Typical examples of these include principal components analysis (PCA) (Abeles and Goldstein Jr, 1977), discrete wavelet transform (DWT) (Letelier and Weber, 2000) and the aforementioned in Section 1. In this work we utilise two methods: First and Second Derivative Extrema (FSDE) and PCA.

\subsubsection{FSDE}

In (Paraskevopoulou et al., 2013), we proposed a hardware-implementable (high accuracy and low computational complexity) feature extraction method that uses derivative-based features. The derivatives are computed as the difference between the current and previous sample points of the spike waveform $(s)$, according to Eq. 1 for the first derivative (FD) and Eq. 2 for the second derivative (SD).

$$
\begin{gathered}
F D(n)=s(n)-s(n-1) \\
S D(n)=F D(n)-F D(n-1)
\end{gathered}
$$

From all the first and second derivatives that were evaluated, highest classification accuracy was shown for the combination of the positive peak of the first derivative $\left(\mathrm{FD}_{\max }\right)$, together with both the negative and positive peaks of the second derivative $\left(\mathrm{SD}_{\min }\right.$ and $\left.\mathrm{SD}_{\max }\right)$. This features combination is referred to as FSDE. Using typical off-line clustering methods, accuracies exceed $85 \%$ have been achieved on typical datasets (Quiroga).

\subsubsection{Principal component analysis}

PCA is considered a gold standard for spike sorting feature extraction. It extracts linearly uncorrelated components from the input signal which are the eigenvectors of the autocorrelation matrix of the original signal, and their number is equal to the number of spike samples. However, the most significant information is contained in the first few. The main disadvantage of the method is its significant computational complexity (Gibson et al., 2008; Paraskevopoulou et al., 2013).

\subsection{Clustering algorithms}

As mentioned, clustering is the final step to separate the spike features into an $m$-dimensional space (Jain, 2010). There are several methods available ( $\mathrm{Xu}$ and Wunsch, 2010) for this that include but are not limited to: $k$-means clustering (Chan et al., 2008), Bayesian clustering (Dai et al., 2008), valley detection (Kim and McNames, 2007; Mahmud et al., 2012), superparamagnetic clustering (Quiroga et al., 2004), expectation maximization (Harris et al., 2000; Pouzat et al., 2002) and artificial neural networks (ANN), $k$-means is one of the most frequent utilised in offline sorting (Gibson et al., 2012). This method is additionally utilised in other applications where hardware-based clustering has been demonstrated (Winterstein et al., 2013).

In this work we focus on clustering methods designed for real-time streaming data. Streaming data clustering is different from traditional static clustering in that it deals with dynamic data where clustering results may 
change or evolve. Due to the massive size of data and restrictions of online systems in memory storage it is also not possible to store large volumes of history, thus methods must rely on short periods (Yogita and Toshniwal, 2012).

In addition there are many challenges associated with streaming data (Khalilian and Mustapha, 2010) including (1) non-supervision (i.e. no knowledge of the number of clusters), (2) tracking cluster evolutions, (3) speed and complexity of computation (including highdimenstionality) and (4) outliers, to name a few. There are several papers that review these challenges and some of the algorithms designed to tackle them (Yogita and Toshniwal, 2012; Khalilian and Mustapha, 2010; Guha et al., 2003).

In general streaming data clustering falls into three categories: hierarchical (connectivity-based), partitionbased and density-based clustering ( $\mathrm{Xu}$ and Wunsch, 2010; Yogita and Toshniwal, 2012).

- Hierarchical clustering employs nested partitions or sub-clusters that build to form a singleton cluster. Here, each cluster can be viewed as the the combination of two clusters from the next lower level or as part of a split cluster from the next higher level. Graphically, a hierarchical clustering algorithm can be viewed as a tree (known as a dendogram) (Steinbach et al., 2000). In the case of agglomerative hierarchical clustering, a popular version of hierarchical clustering, the algorithm is initialised with a certain number of clusters (larger than the anticipated converged outcome). Merging between clusters occurs when the predefined (or adaptively computed) similarity or distance criteria are satisfied.

- Partition-based clustering uses distance metrics to assign data to a section of the clustering space. The most popular of which is $k$-means, which we will describe further as it forms a basis to our own methodology.

- Density-based clustering consider regions of data in the clustering space with a higher density that those outside the cluster region (Xu and Wunsch, 2010). A number of these methods are reviewed in (Yogita and Toshniwal, 2012).

\subsection{1. $k$-means}

$k$-means clustering is a method that aims to partition the spikes (using the selected feature space) into $k$ clusters, in which each spike belongs to the cluster with the nearest mean, as described by Eq. 3 . Hence, $k$ is determined beforehand.

$$
\arg _{S} \min \sum_{(i=1)}^{k} \sum_{\left(x_{j} \in S_{i}\right)}\left\|x_{j}-\mu_{i}\right\|^{2}
$$

where $n$ is the number of spikes, $\left(x_{1}, x_{2} \ldots x_{n}\right)$ is the set of spike features (each of $m$-dimension), $k$ is the number of clusters $(k \leq n)$ and $S_{k}$ are the different sets (i.e. spike classes). $k$-means aims to determine the clusters such as to minimise the sum of squares within each cluster.

An iterative process follows that uses an optimisation process to minimise the sum of squares within each cluster. First, the initial cluster centres are defined as the first $k$ vectors. Second, incoming vectors are classified according to the closest distance (e.g. Euclidean) to the clusters centres. Then, the new centres are calculated as the mean of the newly formed cluster. Finally, the incoming feature vectors are re-classification according to minimum distance until no more change occurs.

This is generally implemented as an iterative algorithm that converges towards the solution. Depending on the feature types (and their dimensionality) the number of iterations required for convergence will vary.

\section{Materials and Methods}

In developing a spike sorting algorithm intended for hardware, the key requirements are to maintain an acceptable accuracy (i.e. lower but still comparable to off-line methods), low complexity (minimal number of computations and low memory requirements) and fully autonomous on-line operation (classification of incoming spikes in 'real-time' and adaptive derivation of number of clusters). In this paper, we propose a novel clustering algorithm, Hierarchical Adaptive Means (HAM) clustering, that encompasses all these attributes.

\subsection{Proposed Method}

The HAM algorithm has two main functions: (1) to assign each spike to a cluster, and (2) to determine the number of clusters. These functions operate in parallel and continually. In assigning an incoming spike to a particular corresponding cluster, the concept is similar to $k$-means (where each spike is assigned to the nearest cluster), only that multiple clusters can then either be merged (total number clusters reduced) or grouped together to provide the final classification. This computation of the number of groups is based on an agglomerative hierarchical algorithm.

The novelty of the proposed algorithm is therefore in combining centroid-based clustering with hierarchical 


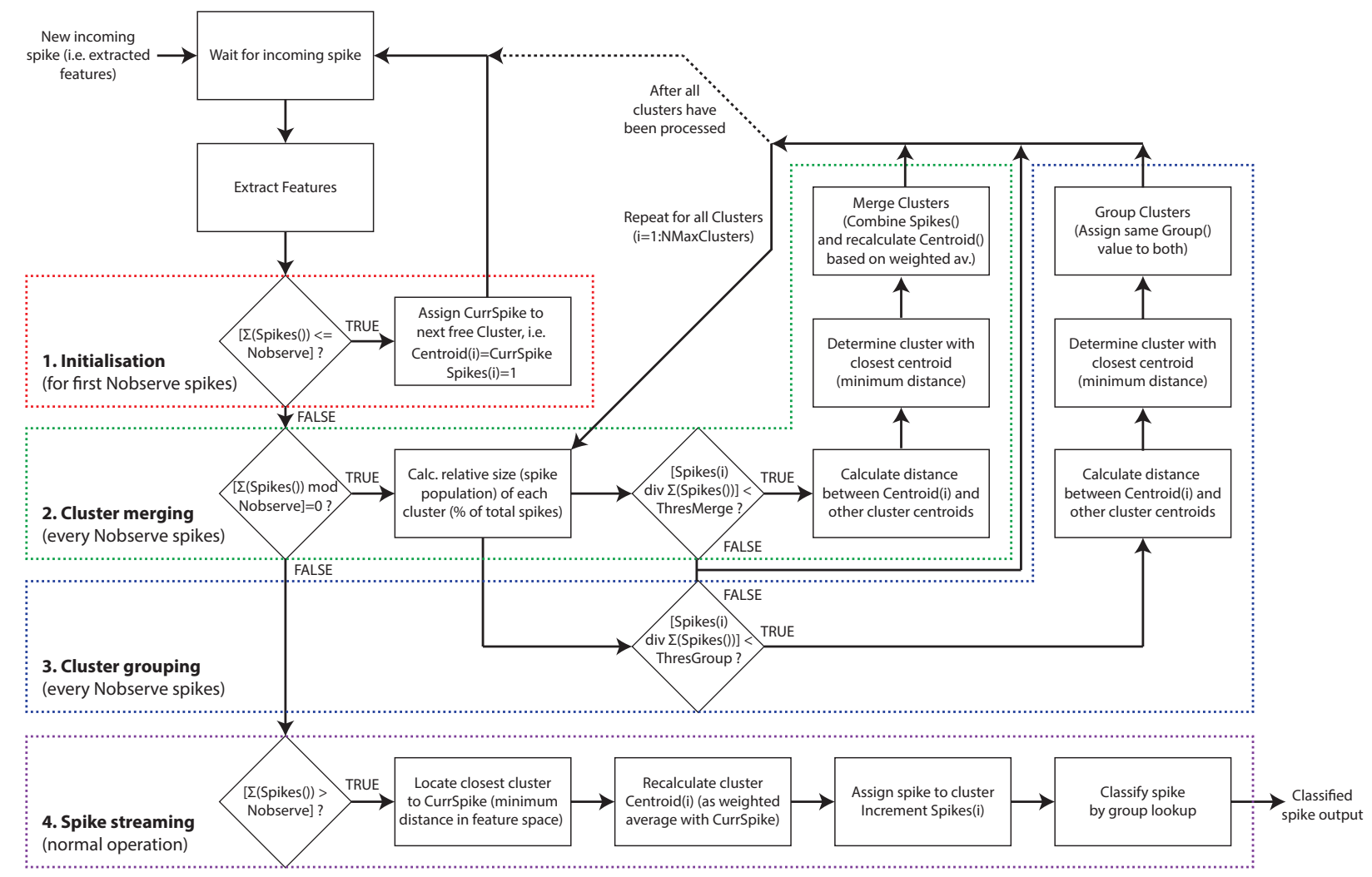

Figure 1: Basic flow chart describing operation of the HAM clustering algorithm.

(connectivity-based) grouping. This achieves a number of attractive features:

- Online processing: There is no requirement for any history (storage of the recorded signal) so any streaming data can be discarded after it is classified. This reduces the amount of memory required.

- Convergence: The clusters can incrementally converge on streaming data (rather than having to iterate through a prerecorded dataset) without compromising the overall classification accuracy. This reduces the amount of computation required compared to traditional centroid-based methods.

- Adaptation: The clusters (and groups of clusters) can evolve or adapt over time and do not require any calibration, training or supervision. This improves the utility in an experimental setting by not requiring any setup time or user intervention.

- Non-uniform Clustering: By grouping multiple centroid-based clusters, the algorithm is able to classify non-uniform combined clusters. For example, in a 3D feature space, this means the algo- rithm isn't constrained to spherical volume as in the case of $k$-means.

The principle of operation of the HAM clustering algorithm is illustrated in Fig. 1. The sections that follow describe the different processes in further detail.

\subsubsection{Algorithm Initialisation}

The algorithm initialises by 'blindly' assigning the cluster centroids to the first $N_{\text {MaxClusters }}$ incoming spikes. The parameter $N_{\text {Maxclusters }}$ is selected to be larger than the maximum possible number of spike classes. With current silicon probes (eg. Blackrock, NeuroNexus, etc), the maximum number of identifiable neurons (i.e. recorded at each electrode) does not surpass 10 (Pedreira et al., 2012), with the average often being only 2 or 3 . Therefore, we have selected $N_{\text {MaxClusters }}=11$.

After this initial cluster centroid assignment, the clusters are continually adjusted through spike streaming (under normal operation) and cluster merging/grouping, so no special training period is enforced. This means that the classification process requires some time (typ- 
ically a few seconds) until its accuracy converges towards a 'final' value. Furthermore, as this initialisation process can occur when the system is powered up, or a system reset occurs, there is no requirement for any non-volatile memory.

\subsubsection{Cluster Assignment}

After the initialisation, each incoming spike is assigned to the closest cluster based on the Euclidean distance between the spike and closest centroid in feature space (Eq. 4).

$$
j_{\text {min }}=\arg \min \sqrt{\sum_{i=1}^{N_{\text {features }}}\left|C_{i, j}-x_{i}\right|^{2}}
$$

where $j_{\min }$ is the assigned cluster, $N_{\text {features }}$ is the number of features, $C_{i, j}$ is the $i^{\text {th }}$ feature of the $j^{\text {th }}$ cluster centroid vector, and $x_{i}$ is the $i^{\text {th }}$ feature of the current spike vector.

On assignment, the cluster centroid is adjusted based on a weighted mean (Eq. 5), and the spike count (i.e. the number of spikes belonging to that cluster) is incremented.

$$
C_{i}=\left(\frac{S_{i}}{S_{i}+1}\right) C_{i}+\left(\frac{1}{S_{i}+1}\right) x
$$

where $S_{i}$ is the total number of spikes assigned to the $i^{\text {th }}$ cluster, i.e. the selected cluster $\left(i=i_{\min }\right)$.

\subsubsection{Cluster Merging and Grouping}

At predefined intervals (for example, every 11 spikes) cluster merging and grouping occur. The difference between these is that the merging process reduces the number of clusters by directly changing the clusters, whereas cluster grouping is connectivity-based and therefore the original cluster data is maintained. The cluster merging is to eliminate small clusters (i.e. any clusters that contain below $10 \%$ the entire spike population), by merging them to their nearest neighbour. The grouping links each cluster to a group (where the number of groups is fewer than the number of clusters) and therefore maintains the individual cluster granularity. This is to associate larger clusters that are in close proximity to each other in feature space to a common group, that ultimately corresponds to the spike class output.

Criterion for Cluster Merging: As the merging process is irreversible, the criteria are stricter than for grouping. The size of each cluster is defined as the number of spikes assigned to it. If the cluster size is smaller than the difference between the standard deviation and mean of all cluster sizes (described in Eq. 6), then the cluster is merged with the nearest cluster.

$\mathrm{S}_{j}<\sqrt{\frac{1}{M} \sum_{i=1}^{M}\left(\mathrm{~S}_{i}-\frac{1}{M} \sum_{i=1}^{M} \mathrm{~S}_{i}\right)^{2}}-\frac{1}{M} \sum_{i=1}^{M} \mathrm{~S}_{i}$

where $S_{j}$ is the the size of cluster $j$, and $M$ is the total number of clusters.

Cluster Merging: To determine the nearest cluster, the minimum Euclidean distance is calculated between the centroid of the cluster to be merged $C_{j}$ and all the other cluster centroids $C_{i}(i=1: M)$. This is calculated as in spike assignment (Eq. 4). The centroid of the new merged cluster is then calculated as a weighted mean (Eq. 7).

$$
C_{i}=\frac{S_{i} C_{i}+S_{j} C_{j}}{S_{i}+S_{j}}
$$

where $C_{i}$ and $C_{j}$ are the centroids, and $S_{i}$ and $S_{j}$ are the respective sizes of the two clusters (i.e. clusters $i$ and $j$ ) being merged.

Cluster Grouping: This begins by calculating the cluster size ratio of each of the clusters. This is defined as the ratio of the size of each cluster divided by the total number of spikes. If this ratio is less than 0.2 (i.e. less than $20 \%$ the total spikes) then the cluster is eligible for grouping (Eq. 8.

$$
\frac{S_{i}}{\sum_{i=1}^{M} S_{i}}<0.2
$$

where $S_{i}$ is the the size of cluster $i$ and $M$ is the total number of clusters.

Clusters are grouped only to their mutually nearest clusters (i.e. clusters whose centroids are closest to each other). This is repeated until the grouping criterion is no longer satisfied.

\subsubsection{Spike Classification}

For each spike received, a group lookup is performed to classify the spike. As the cluster merging and grouping occurs only once every $N_{\text {observe }}$ spikes, the group lookup is based on the current definitions at the time the new incoming spike is received.

\subsubsection{Memory and Computational Requirements}

As previously mentioned, a key feature of the proposed algorithm is the low complexity, both in terms of memory and computation, and is thus ideally suited for future hardware implementation. 
Table 2: Memory requirements for the HAM clustering algorithm (per incoming spike stream, i.e. recording channel)

\begin{tabular}{|c|c|c|c|c|}
\hline Name & Symbol & Description & Type & Bytes \\
\hline \multicolumn{5}{|c|}{ (Global static constants, i.e. ROM) } \\
\hline $\mathrm{N}_{\text {Observe }}$ & $N$ & Observation period (for re-evaluating clusters/groups) & int8 & 1 \\
\hline $\mathrm{N}_{\text {MaxClusters }}$ & $M$ & Maximum number of clusters (currently $N=M=11$ ) & int4 & 0.5 \\
\hline $\mathrm{N}_{\text {Features }}$ & $F$ & Number of features (currently $F=2$ ) & int4 & 0.5 \\
\hline \multicolumn{5}{|c|}{ (State variables, i.e. RAM) } \\
\hline$i, j$ & $i, j$ & Temporary variables & int8 & 1 \\
\hline Threshold $_{\text {Merge }}$ & $T_{M}$ & Threshold for cluster merging & float32 & 4 \\
\hline Threshold Group & $T_{G}$ & Threshold for cluster grouping & float32 & 4 \\
\hline CurrSpike $\left[1: \mathrm{N}_{\text {Features }}\right]$ & $x_{i}$ & Current spike features (vector of $F$-elements) & float32 & $4 F$ \\
\hline Centroid[1: $\left.\mathrm{N}_{\text {Features }}\right]\left[1: \mathrm{N}_{\text {MaxClusters }}\right]$ & $C_{i, j}$ & Cluster centroids (array of $F \times M$-elements) & float32 & $4 F \cdot M$ \\
\hline Group $\left[1: \mathrm{N}_{\text {MaxClusters }}\right]$ & $G_{i}$ & Cluster group assignments (vector of $M$-elements) & int4 & $0.5 M$ \\
\hline Spikes[1: $\left.\mathrm{N}_{\text {MaxClusters }}\right]$ & $S_{i}$ & No. of spikes in cluster (vector of $M$-elements) & int16 & $2 M$ \\
\hline
\end{tabular}

Table 3: Computation required for the HAM clustering algorithm (basic ALU instructions)

\begin{tabular}{|c|c|c|c|c|c|}
\hline Process & Memory accesses & Conditions & Add/Subtracts & Multiply/divides & Other \\
\hline \multicolumn{6}{|c|}{ (For first M spikes) } \\
\hline Initialisation & 4 & 1 & 1 & - & - \\
\hline \multicolumn{6}{|c|}{ (Normal operation - for every spike) } \\
\hline Spike assignment & $F \cdot M$ & M & $(F+1) M$ & $F \cdot M$ & $M \sqrt{ }$ \\
\hline Centroid re-calculation & $4 F$ & - & $F$ & $2 F$ & - \\
\hline Adjust and group lookup & $(F+4)$ & - & 1 & - & - \\
\hline \multicolumn{6}{|c|}{ (Cluster re-evaluation - every $\mathbf{N}_{\text {Observe }}$ spikes) } \\
\hline Merge criterion $\dagger$ & $3 M$ & M & $3 M$ & $(M+2)$ & $1 \sqrt{ }$ \\
\hline Merge clusters $* \dagger$ & {$[(M+8) F+1]$} & $2 M$ & {$[(F+1) M+3 F+1]$} & $(M+6) F$ & $M \sqrt{ }$ \\
\hline Group criterion/assignment & $(2 M+1)$ & $M$ & $M$ & - & - \\
\hline
\end{tabular}

$\dagger$ Merge process only occurs during first $10 N_{\text {Observe }}$ periods, * Occurs only once per merge (since irreversible)

Memory/Data: Unlike the vast majority of spike sorting methods, the proposed algorithm does not require any signal history nor buffer memory. The only memory requirements are the state variables for computing and tracking the cluster centroids and groups. Furthermore, as the algorithm is completely adaptive and selfinitialising, the state variables can be implemented using volatile memory (i.e. RAM). A list of the variables required are given in Table 2.

The amount of memory required per incoming spike stream, i.e. recording channel, is given by Eq. 9 .

$$
N_{\text {bytes }}=[9+4 F+M(4 F+2.5)]
$$

where $N_{\text {bytes }}$ is the memory requirement (per channel), $F$ is the dimensionality of the feature space and $M$ is the maximum number of clusters. For example, in a
3D feature space with a maximum of 10 clusters, the memory required would be 166 bytes per channel.

Computation: The processes required for this algorithm can be divided into three main categories (based on when they are in operation). These are: (1) initialisation; (2) cluster re-evaluation (merging and grouping); and (3) spike streaming (normal operation). Initialisation occurs only for the first $N_{\text {initial }}$ incoming spikes, whereas cluster re-evaluation occurs once for every $N_{\text {observe }}$ spikes. Spike streaming, however, occurs continuously, i.e. for every incoming spike. The amount of computation required for each sub-process (based on basic ALU instructions) has been estimated in Table 3. Therefore, the number of computations per incoming spike, can be determined by Eq. 10 . 


$$
\begin{aligned}
I_{\text {streaming }} & =(3 M+8) F+42 M+5 \\
I_{\text {cluster }} & =(4 M+1) \\
I_{\text {average }} & =I_{\text {streaming }}+\frac{I_{\text {cluster }}}{N_{\text {observe }}}
\end{aligned}
$$

where $I_{\text {streaming }}$ and $I_{\text {cluster }}$ are the number of instructions per spike streaming and cluster re-evaluation computation respectively. The average computational load per incoming spike ( $I_{\text {average }}$ ) can then be expressed by distributing $I_{\text {cluster }}$ over the observation period $\left(N_{\text {observe }}\right)$, excluding initialisation and cluster merging (that are essentially one-off operations). These expressions assume all ALU instructions require the same processing time, except square root ( $\approx 40$ instructions).

For example, the average computational load for a $3 \mathrm{D}$ feature space with a maximum of 10 clusters, would be $\approx 543$ instructions/spike. Assuming 3 units, each with firing rate of 25 spikes/s, this would correspond to 0.04 MIPS. For low power processors (eg. ARM Cortex-M0+ consumes $11.21 \mu \mathrm{W} / \mathrm{MHz}$ ), this corresponds to $<10 \mu \mathrm{W} /$ recording channel (to achieve real-time, adaptive spike clustering).

\subsection{Test datasets}

The proposed clustering algorithm has been tested with both synthetic and more realistic data based on real recordings. The synthetic data firstly allows us to test the algorithm under different conditions (for example, with different spike shapes and at multiple noise levels) with a known ground truth. This is essential in correctly quantifying classification accuracy and benchmarking to other methods. The data based on recordings data then allows us to validate the algorithm under more realistic conditions (i.e. including instrumentation noise and measured background activity), however with less flexibility than with simulated data.

The clustering difficulty (ability of clustering algorithm to separate spikes from different origin) depends on the SNR of each input signal and the similarity between the spike shapes in each dataset. To quantify similarity, the Bray-Curtis similarity index (Lian et al., 2010) is used, as defined by Eq. 11.

$$
S_{x, y}=1-\frac{\sum_{i=1}^{N}|x(i)-y(i)|}{\sum_{i=1}^{N}|x(i)|+|y(i)|}
$$

where $x, y$ are the 2 spike waveforms being compared and $N$ is the number of sample points. $S_{x, y}$ is in the range $(0-1)$, with 1 corresponding to identical signals.

\subsubsection{Synthetic test data}

The specific datasets, originally used in (Quiroga et al., 2004) to evaluate the spike sorting accuracy with wavelets and superparamagnetic clustering, are publicly available (Quiroga). These datasets have been generated by randomly selecting spike signals from a database (based on over 500 recordings from the human neocortex and basal ganglia) with noise added by superimposing random (scaled) spike signals to emulate the neuronal background activity (spikes generated by neurons at a proximity of between 50 and $140 \mu \mathrm{m}$ from the electrode (Buzsaki, 2004)). Although these datasets include this emulated biological noise, real recordings would also include thermal noise induced by the electrodes and recording electronics (Shoham and Nagarajan, 2004).

For the simulations in this paper, we are using four of these datasets (originally named Easy1, Easy2, Difficult 1 and Difficult2). Each of the datasets contains 3 different types of spikes shapes and 4 different noise levels (with standard deviations of 0.05, 0.01, 0.15, and 0.2).

For each of the simulated datasets, the similarity measure between all three spike templates in a dataset is calculated and presented along with mean spike waveforms in Fig. 2a. The mean spike waveforms (for each of the 3 classes in the 4 test datasets) have been generated by taking a mean across all spikes in each cluster.

\subsubsection{Realistic test data}

The signals used were based on recordings from the primary motor cortex of a female macaque monkey using Teflon-insulated tungsten microwire electrodes of $50 \mu \mathrm{m}$ diameter and approximately $100-200 \mathrm{kOhm}$ impedance. Details of the electrode array can be found in (Jackson and Fetz, 2007). The signals were amplified using a Multichannel Systems MP81 headstage $(\times 10$ gain) and PGA1632 amplifier ( $\times 1000$ gain and $300 \mathrm{~Hz}-$ $8 \mathrm{kHz}$ ) and recorded using a CED Power 1401 data acquisition system (sampled at $100 \mathrm{kHz}$ ). The test dataset (shown in Fig. 2b) was then constructed by combining 3 recordings, each containing the spiking activity of a different single unit. This was achieved by extracting the mean spike templates from the first two recordings and embedding these into the third recording at intervals corresponding to spike rates of $18 \mathrm{~Hz}$ and $20 \mathrm{~Hz}$ respectively. In this manner the test dataset has three distinct spike clusters that are known but also realistic background activity. Each of these 3 classes contains 959,1198 , and 1021 spikes respectively.

\subsection{Classification accuracy}

The proposed HAM clustering algorithm is adaptive and therefore the classification accuracy is expected to 


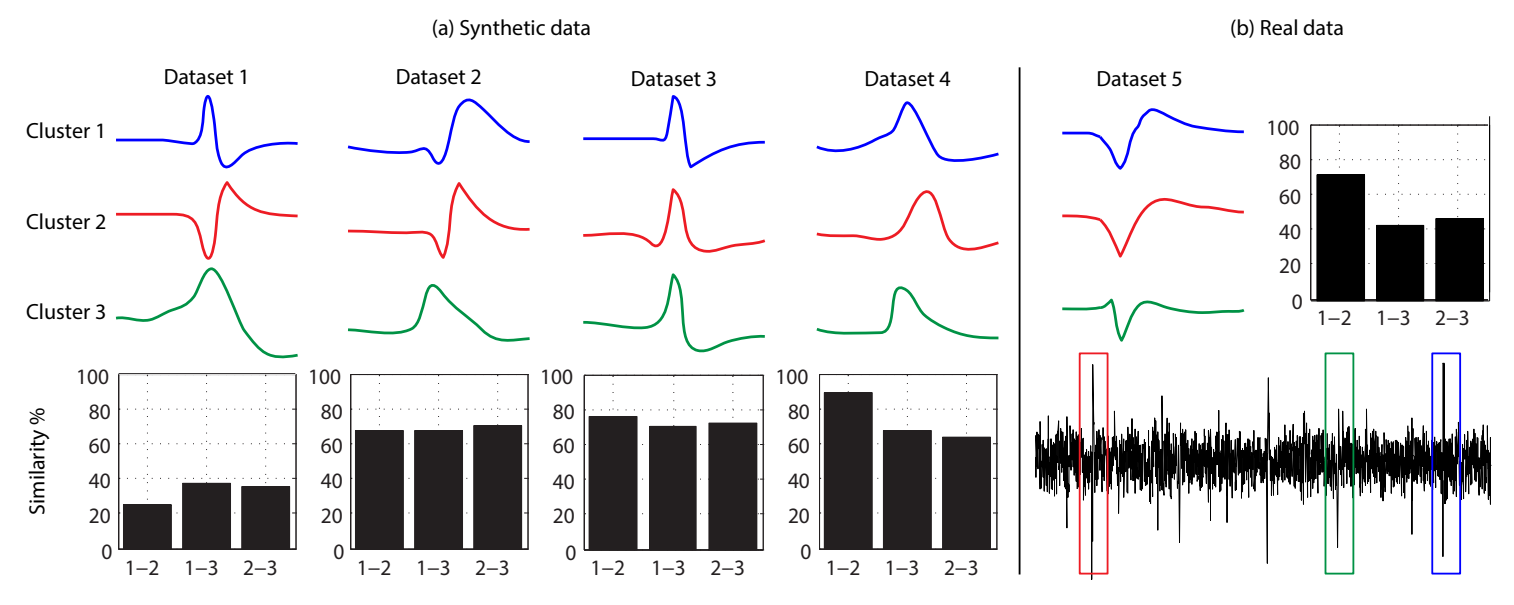

Figure 2: Test datasets used showing the mean, peak-aligned spike classes with corresponding degrees of separability (using Bray-Curtis similarity index) for the (a) synthetic; and (b) real data (also showing a sample segment of the signal)

increase converging towards a steady-state value. This is quantified by defining the incremental accuracy $(I A)$ measure. This is computed for every incoming spike, defined as the ratio of the number of correctly classified spikes over the total number of spikes (Eq. 12).

$$
I A_{j}=\frac{\sum_{i=1}^{j} C S}{j}
$$

where $I A_{j}$ is the incremental accuracy of $j$-th spike and $C S$ is the number of correctly classified spikes.

Another important aspect of the algorithm is that the clusters and groups are re-evaluated at fixed intervals (i.e. observation period of $\left(\mathrm{N}_{\text {observe }}\right)$ spikes). To quantify whether the algorithm is converging towards a correct clustering output at each renewal, a moving accuracy $(M A)$ measure is introduced (Eq. 13), computed every $\mathrm{N}_{\text {observe }}$ spikes.

$$
M A_{j}=\frac{\sum_{i-N_{\text {observe }}}^{i} C S}{N_{\text {observe }}}, \text { with } j=\frac{i}{N_{\text {observe }}}
$$

where $\mathrm{MA}_{j}$ is the moving accuracy of the $j \cdot N_{o b s e r v e}^{\text {th }}$ spike, $\mathrm{N}_{\text {observe }}$ is the observation period, and $C S$ is the number of correctly classified spikes.

\section{Results and Discussion}

\subsection{HAM Clustering on Simulated Neural Signals}

In this Section, the HAM clustering algorithm is tested using the simulated data previously described. In these tests, FSDE is used for feature extraction (as described in (Paraskevopoulou et al., 2013)) with $N_{\text {features }}=3(=F)$.

\subsubsection{Initialisation and Cluster Evolution}

As described previously, there is an initial phase whereby the first $M_{\text {MaxClusters }}(=11)$ spikes each assigned to separate clusters. Thereafter each incoming spike is assigned to the closest cluster and cluster centroid is adjusted. Furthermore, every $N_{\text {observe }}(=11)$ spikes, the clusters are re-evaluated and if certain criteria are satisfied are merged and/or grouped. This entire process is illustrated in Fig. 3. Here, a portion of dataset 3 (at $0.02 \sigma$ noise level) containing 55 spikes corresponding to approximately $1 \mathrm{~s}$ is used (data segment shown in Fig. 3a). The state of the HAM clustering algorithm is then shown every $N_{\text {observe }}$ spikes showing the spike assignment (Fig. 3b), cluster merging and grouping (Fig. 3c), and final spike classification (Fig. 3d).

In this illustrated example, after the first $N_{\text {observe }}$ period (i.e. the initialisation phase), no classification decision has been yet made. However, the algorithm quickly converges. After the second $N_{\text {observe }}$ period (i.e. after 22 spikes), it can be observed that the algorithm correctly classifies the spikes (and clusters) into 3 groups. The incremental accuracy (shown in Fig. 3e) thus reaches above $90 \%$. However, although the spikes appear to have been correctly classified, the clusters continue to form and regroup until the fourth $N_{\text {observe }}$ period (i.e. 44 spikes). The clusters (and groups) converge thereafter.

\subsubsection{Steady-state Clustering}

The steady-state classification of the HAM clustering algorithm can be observed when testing with a larger dataset; such that the clusters converge towards a steady 
(a) Test dataset 3

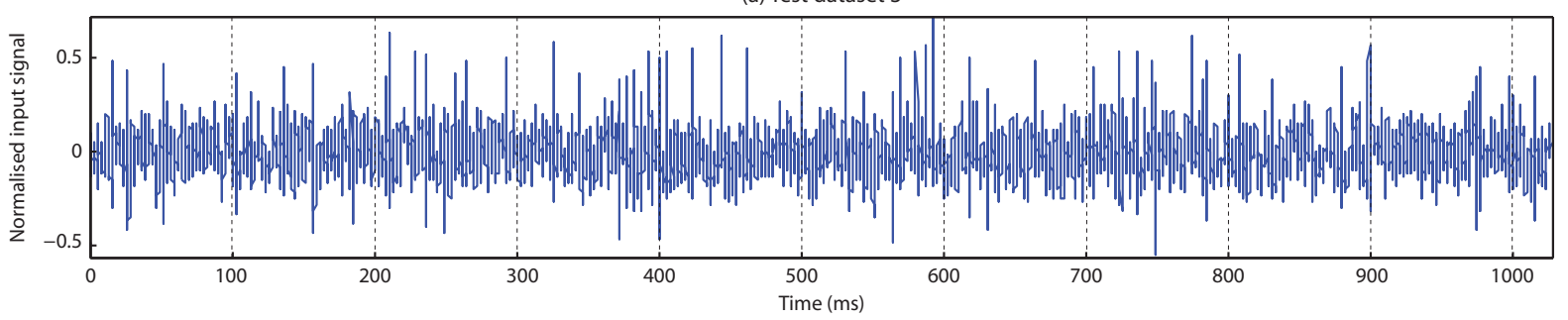

(b) Spike assignment and centroid tracking

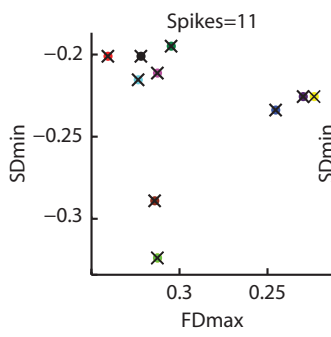

Spikes $=33$
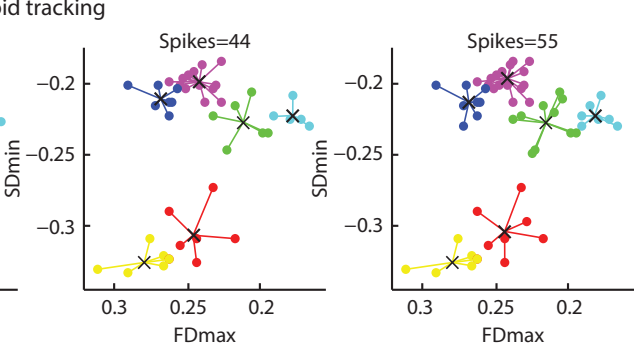

(c) Cluster re-evaluation (merging and grouping)
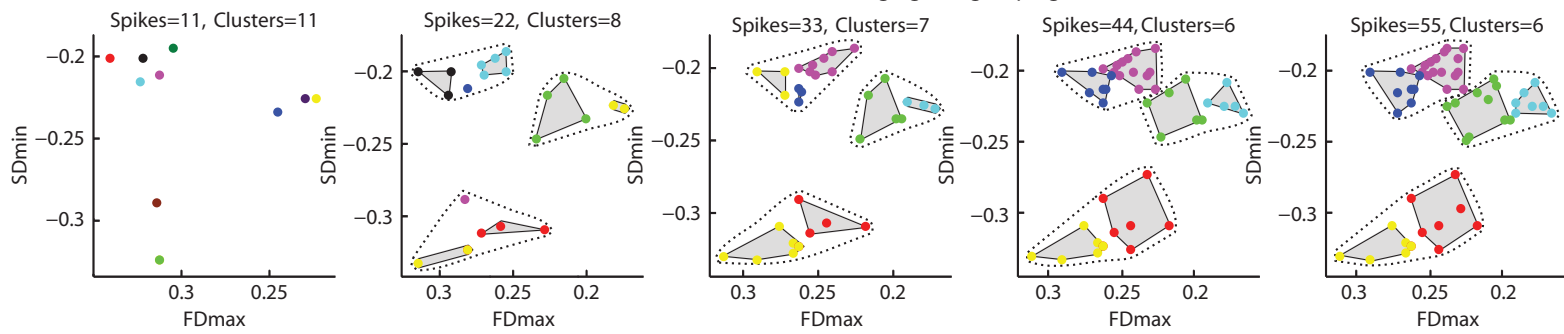

(d) Final spike classification (group lookup)
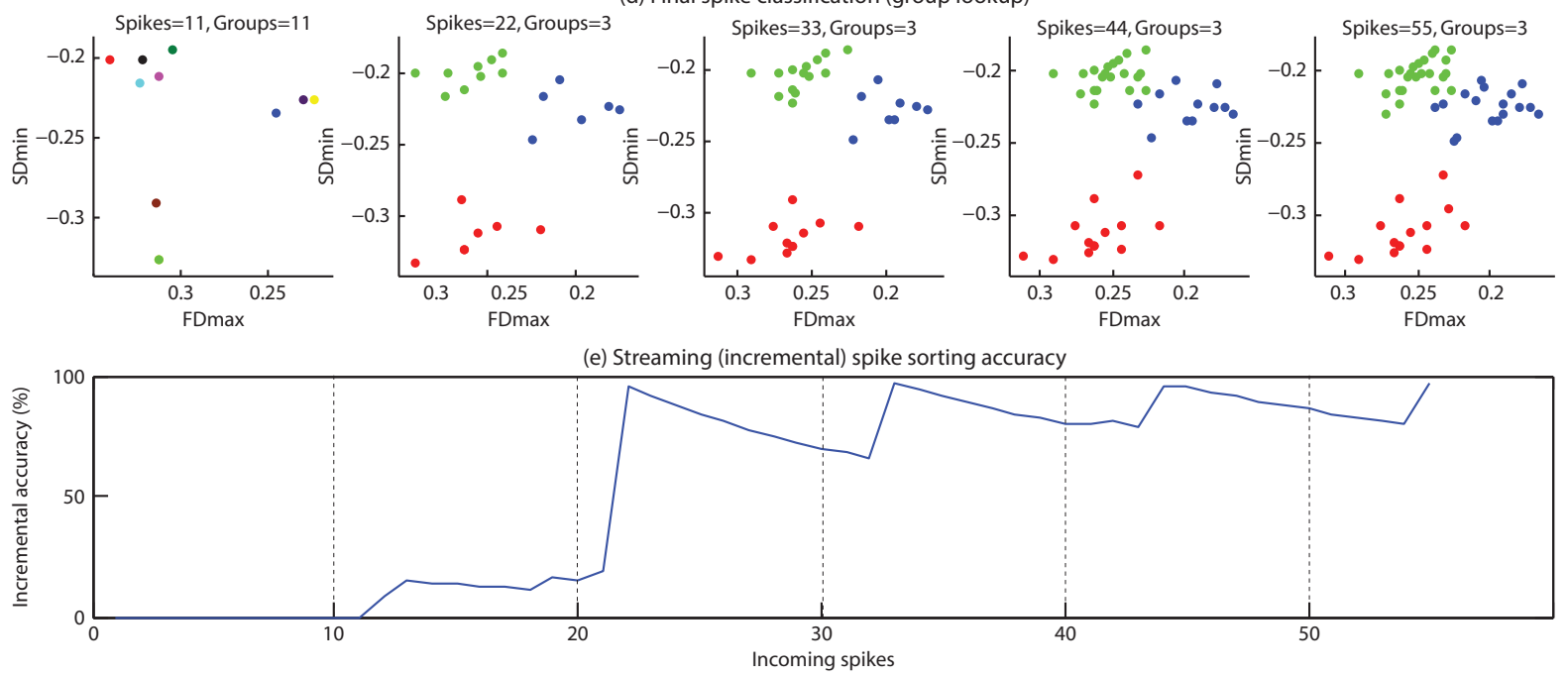

Figure 3: Initialisation of the HAM clustering algorithm. (a) Initial segment of dataset $3(0.2 \sigma$ noise level); (b) spike assignment (to their respective clusters) showing recalculated centroids; (c) cluster evolution and grouping; (d) final spike classifications (after grouping) and (e) Incremental accuracy. FSDE is used for feature extraction (showing only $\mathrm{FD}_{\max }$ and $\mathrm{SD}_{\min }$ in plots for clarity) and each cluster and spike class are represented with a different colour. 
state. Fig. 4 shows the output of the HAM clustering algorithm (illustrating the clusters, centroids and groups) compared to the ground truth for the 4 (synthetic) test datasets (at the highest noise level). All these results were obtained for an $M_{\text {MaxClusters }}$ and $N_{\text {observe }}$ of 11 .

It can be observed that in the ground truth (Fig. 4c), some of the points (with each point corresponds to a spike feature vector) seem erroneous. For example, in dataset 1 , some spikes that are assigned to cluster 2 (green colour) appear within the expected region for cluster 3 (red colour). This is because of overlapping spikes, i.e. when features belonging to different spikes are captured within the same window (during feature extraction). HAM clustering bases its classification decision on these feature vectors, thus this will add to the classification error. We chose not to eliminate overlapping spikes from datasets for more realistic evaluation of the algorithm. To date, there have been no reported online methods for dealing with overlapping spikes that have the potential of efficient hardware implementation.

\subsubsection{Classification Accuracy}

In computing the incremental accuracy (shown in Fig. 5a), it can be observed that the algorithm successfully converges to the correct number of spike classes with accuracies of approximately $90 \%$ (for a noise level of $0.1 \sigma)$. .

Similarly the moving accuracy (shown in Fig. 5b) is generally high (over 90\%). However, fluctuations in the moving accuracy value can also be observed. These are more evident in the datasets with higher similarity between clusters (e.g. in dataset 4 the moving accuracy can drop down to $10 \%$ ). This decrease in moving accuracy signifies that during that observation period, HAM is not converging to the correct number of clusters. When the $N_{\text {observe }}$ period is low, there will be periods where only two clusters dominate, and as such the third cluster may not be identified and classified. In the datasets tested, this occurs very infrequently and thus does not affect the overall classification accuracy.

\subsubsection{Comparison to k-means Clustering}

To benchmark the performance of HAM clustering, we compare it to the offline/iterative method it was derived from, i.e. $k$-means. To ensure that $k$-means converges for all datasets and noise levels, 10-iterations are used, with the number of clusters being predefined. Hence, we note that the HAM clustering algorithm here is being compared to a significantly more computationally demanding method. Furthermore, we have tested both methods (HAM clustering and $k$-means) using both FSDE and PCA features, and for all datasets and noise levels. The results are shown in Fig. 6. In the case of HAM clustering, the classification accuracy has been quantified as the average of the moving accuracy (including the initialisation phase).

HAM clustering generally shows a comparable (almost identical) accuracy to $k$-means. For FSDE features, HAM clustering shows a reduced accuracy only in dataset 2 by approximately $10 \%$. For PCA features, HAM clustering accuracy decreases more rapidly than $k$-means in dataset 3 for increasing noise levels, although both show similar accuracies at the highest noise level. These results confirm that the proposed classification algorithm achieves a comparable accuracy to a commonly used iterative method. Moreover, HAM clustering, unlike $k$-means, automatically determines the number of clusters. Finally, an important observation is that both classification algorithms show similar behaviour for each choice of feature vectors. This proves the robustness and versatility of the HAM clustering algorithm (i.e. it can be used with different features without compromising the classification accuracy).

\subsection{HAM clustering on realistic neural signals}

For testing the HAM clustering method on our realistic test dataset (Fig. 2b), we used PCA features (Harris et al., 2000). This is because this dataset has a high level of environmental noise and separation via FSDE features deteriorates with high levels of white noise. As such, neither HAM clustering, nor $k$-means could separate this data using FSDE-features. The results using PCA features are shown in Fig. 7. This demonstrates that the accuracy of HAM clustering is very close to that of $k$-means, achieving an accuracy of $95.25 \%$ compared to $96.22 \%$.

\subsection{Discussion}

We start by comparing the main characteristics of HAM clustering to commonly used clustering methods for spike sorting ( $k$-means, SPC, Osort and KlustaKwik) in Table 4. HAM clustering deals with all the challenges of data streaming. It presents high accuracy, has low memory requirements and low computational complexity, is unsupervised (i.e automatically computes the number of clusters), tracks the evolution of clusters in real-time and requires a minimal number of usertuneable parameters.

However, despite the many attractive features, there are a number of issues that need to be addressed to ensure an efficient and robust hardware implementation. 

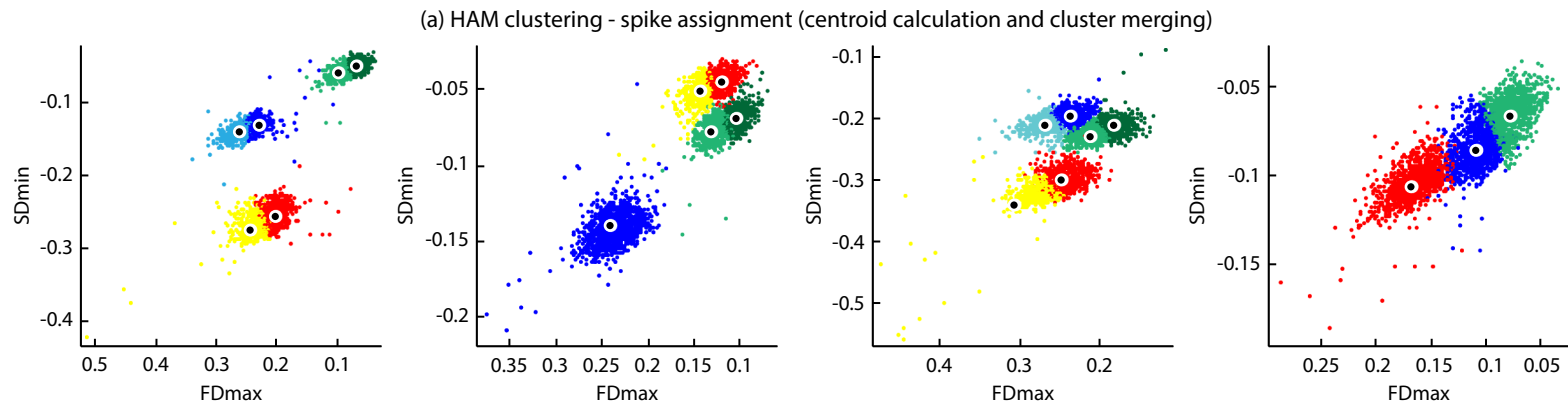

(b) HAM Clustering - classification (cluster grouping)
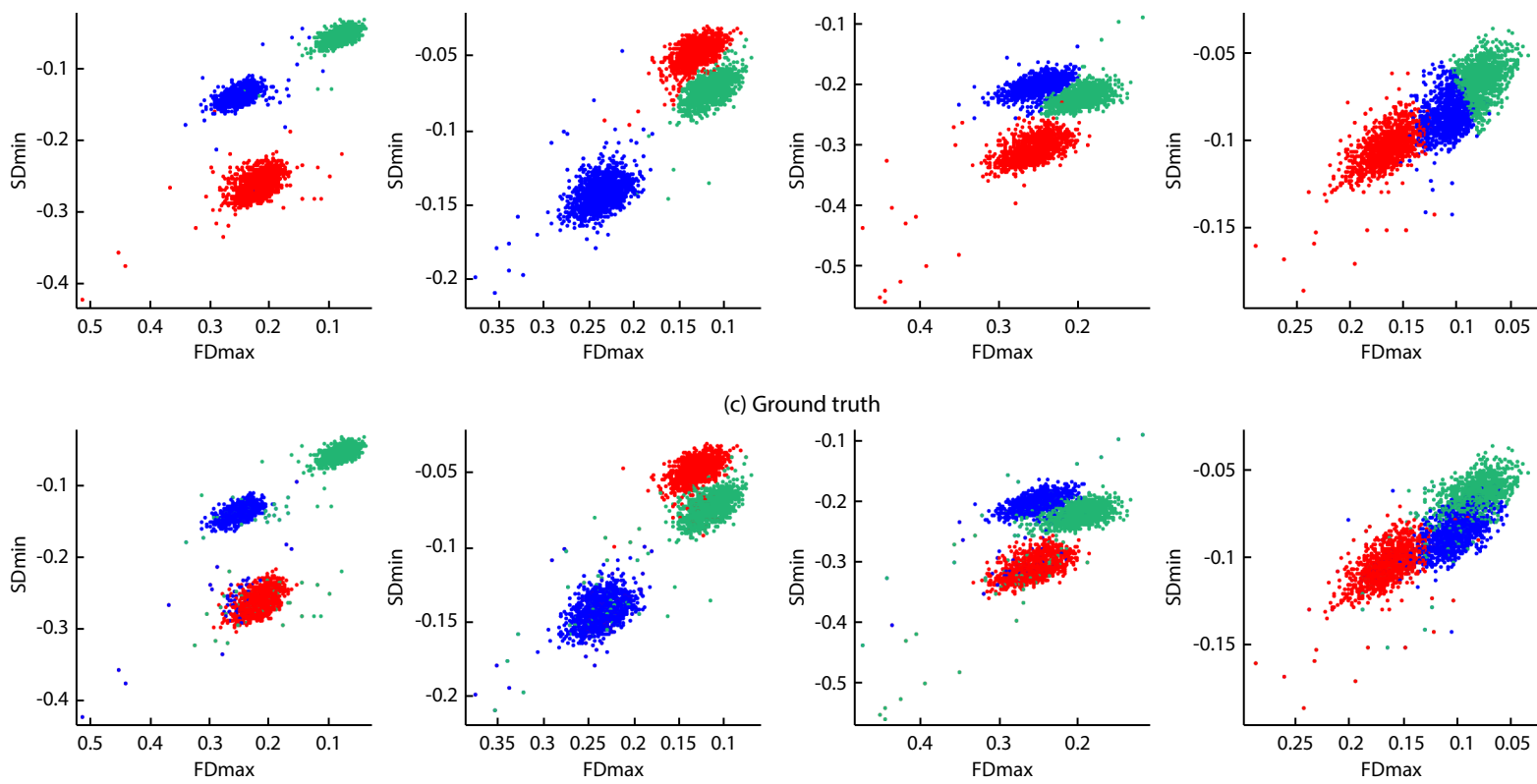

(c) Ground truth
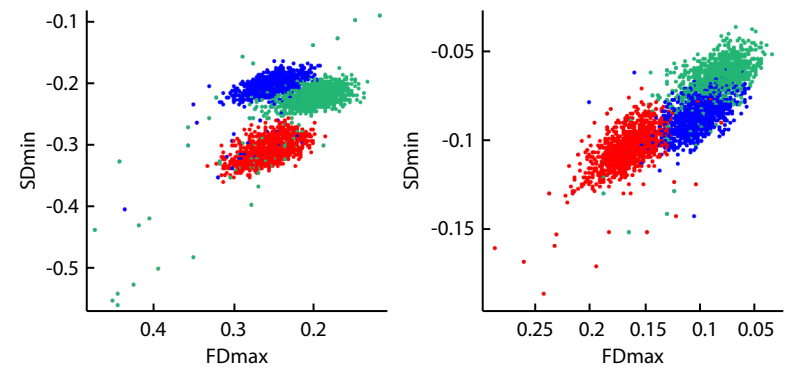

Figure 4: Spike classification of the test datasets using FSDE features (showing only $\mathrm{FD}_{\max }$ and $\mathrm{SD}_{\min }$ in plots for clarity). Shown are: (a) HAM clustering spike assignment with cluster centroids; (b) final classification after grouping with HAM clustering; and (c) the actual clusters (as specified in each dataset at an noise level of 0.2 sigma).

The following sections discuss specifically the classification accuracy, computational complexity and adaptability.

\subsubsection{Classification accuracy}

The need for a high and sustained classification accuracy is essential. In all our results presented herein, the initial (or maximum) number of clusters $\left(\mathbf{M}_{\text {MaxClusters }}\right)$ and the observation period ( $\left.\mathrm{N}_{\text {Observe }}\right)$ are set at 11 . This was not arbitrarily selected, but based on the fact that the maximum number of neurons that can be observed at each electrode is 10 . Given these parameters, HAM clustering shows high accuracy for all the datasets we have tested. However, a key observation we have made is that the algorithms performance is sensitive to this pa- rameter selection. This is evident in Fig. 8, showing the classification accuracy of HAM on simulated data for varying $\mathbf{M}_{\text {MaxClusters. }}$.

These results show that the choice of $\mathrm{N}_{\text {MaxClusters }}$ can affect the classification accuracy. In all cases, for consistency and to ensure proper initialisation of the algorithm, $\mathrm{N}_{\text {observe }}$ has the same value as $\mathrm{M}_{\text {MaxClusters. }}$. At each of the tested noise levels, the classification accuracy has been averaged over the four datasets. The results suggest that a different $\mathrm{M}_{\text {MaxClusters }}$ can reduce the classification accuracy for certain datasets at certain noise levels. This inconsistency needs to be further investigated. 

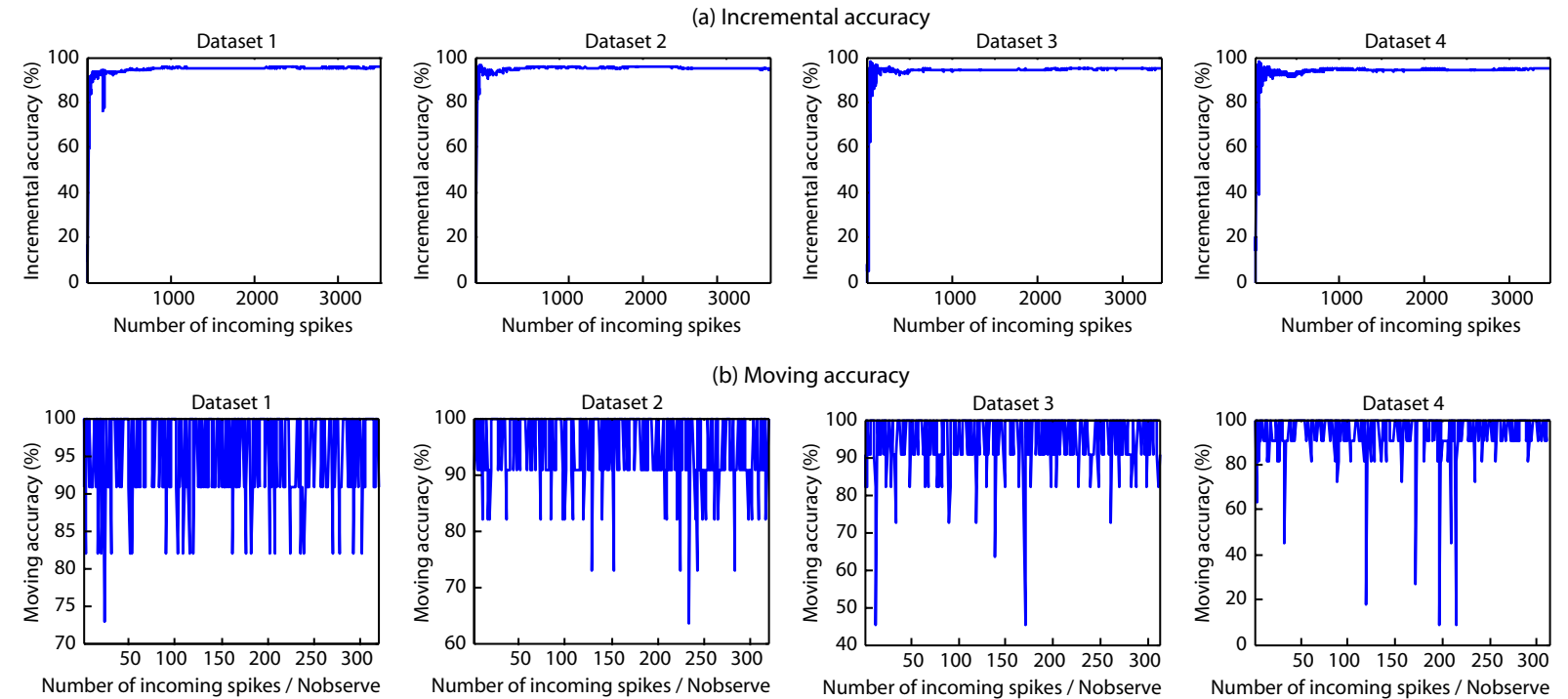

Figure 5: Accuracy of HAM clustering using FSDE features for all datasets (noise of $0.1 \sigma$ ). Shown are: (a) incremental accuracy; (b) moving accuracy.
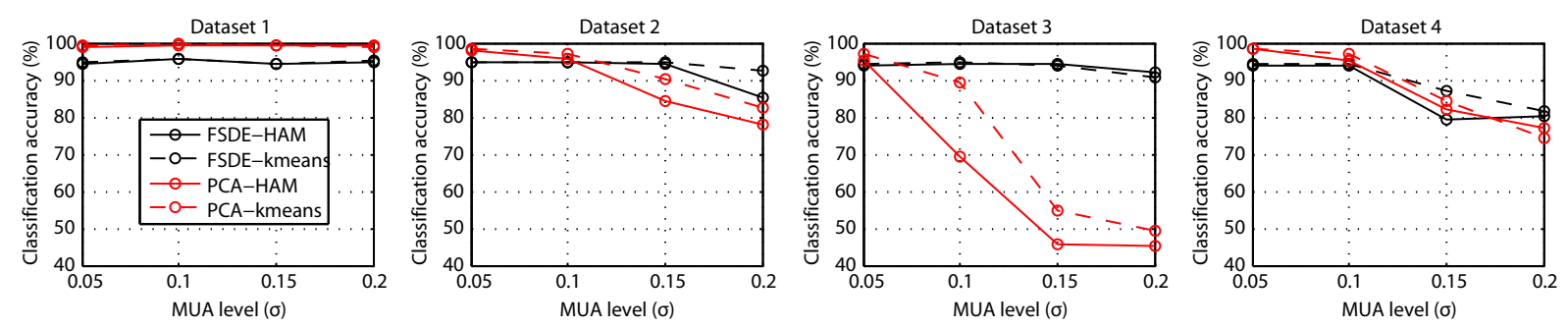

Figure 6: Comparative classification accuracies of HAM clustering and $k$-means (for both FSDE and PCA features).
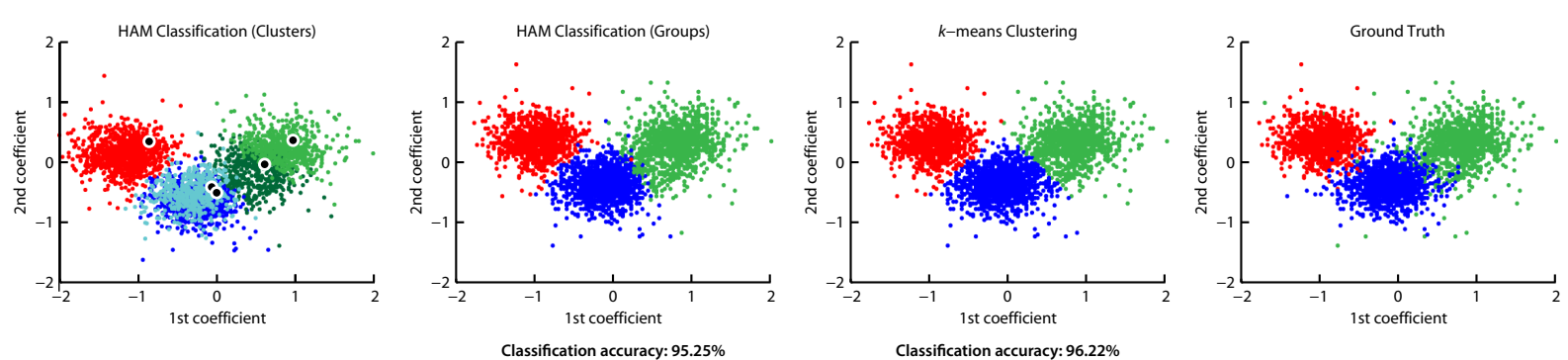

Figure 7: Clustering results for our realistic test dataset using PCA features. Shown are (from left to right): (a) HAM clustering spike assignment (to cluster centroids); (b) HAM clustering output (after grouping); (c) clustering via 10-iterations $k$-means, (d) ground truth.

\subsubsection{Computational complexity}

Given the stringent requirements imposed by implantability (i.e. power, bandwidth and volume) (Eftekhar et al., 2010), computational complexity is as important as classification accuracy in developing a viable platform.

Although the algorithm has been developed with both the computational complexity and memory re- quirements in mind, further optimisation is necessary once a hardware (computational) platform has been selected. Specific points that need to be addressed are:

- All computation (and variables) should be translated from floating to fixed-point.

- Variable datatypes need to be selected based on minimum required precision for each quantity. 
Table 4: Comparison of HAM clustering to state-of-the-art clustering algorithms used in spike sorting (Paraskevopoulou et al., 2013; Wild et al., 2012; Gibson et al., 2012)

\begin{tabular}{c|c|c|c|c|c}
\hline \multirow{2}{*}{ Characteristics } & \multicolumn{5}{|c}{ Clustering Algorithms } \\
& $k$-means & SPC & Osort* & KlustaKwik & HAM \\
\hline Classification accuracy & High & High & Moderate & High & High \\
Computational complexity & Moderate $\dagger$ & High & Low & Moderate & Low \\
Memory requirement & Moderate & Moderate & High & Moderate & Low \\
Unsupervised & No & Yes & Yes & Yes & Yes \\
Real-time/online & No & No & Yes & No & Yes \\
User-tuneable parameters & 2 & 20 & 2 & 10 & 2 \\
\hline
\end{tabular}

*Template matching is used for clustering.

$\dagger$ Taking into account that the algorithm requires multiple iterations to converge.

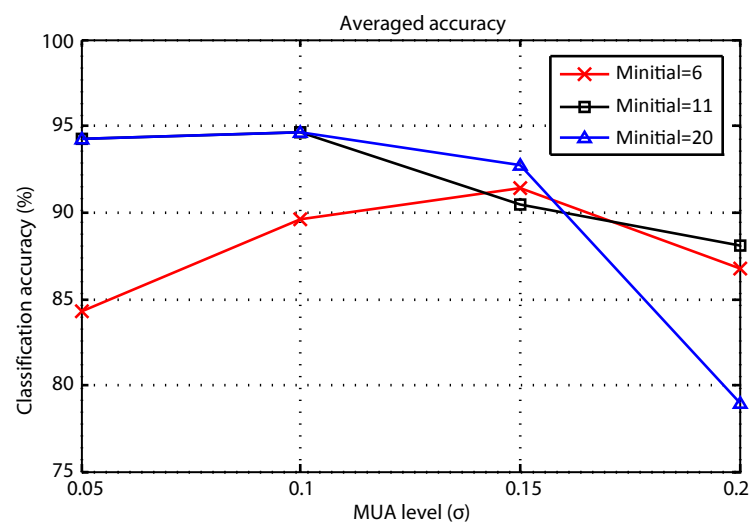

Figure 8: Classification accuracy averaged over all simulated datasets for varying $\mathbf{M}_{\text {initial }}$. In all cases $\mathrm{N}_{\text {observe }}$ is set at the same value as $\mathrm{M}_{\text {initial }}$.

- Efficient implementation of key functions, for example, Euclidean distance measure requires the square and square root functions.

- Reducing complexity of arithmetic operators, for example, wherever possible, replacing multiple/divides by logical left/right shifts (divide/multiple by powers of 2).

Furthermore, further algorithm optimisation is required to reuse variables and share operations wherever. For example, the grouping and merging processes are very similar, as are their criteria. At the moment, they occur independently (i.e. the grouping criteria are checked after merging is complete). To reduce the computational complexity of HAM clustering, grouping and merging should be more efficiently combined.

\subsubsection{Adaptability}

It is common for application specific clustering algorithms (e.g. SPC) to use empirical factors. In HAM, the grouping criterion threshold is empirically derived. This factor has been chosen to yield high classification accuracy for a variety of neural datasets (both simulated and real recordings). Nevertheless, to ensure ubiquitous application of HAM clustering, it should be replaced with an adaptive threshold (like the merging criterion threshold).

Moreover, HAM clustering is based on agglomerative hierarchical clustering starting with a large number of clusters and converging to the optimal number of clusters. This cluster evolution does not take into account possible electrode drift and deterioration of the quality of the recordings for chronically implanted microelectrode arrays (Lebedev and Nicolelis, 2006). In order, to overcome this (allowing for new clusters to form) the algorithm can be reset (i.e. set the number of clusters to initial and clear the cluster size counter) and retrain itself periodically. Another option, is to allow the algorithm to perform both merging and splitting functions. That is, when an incoming spike is located "too far" from the centroids of existing clusters, a new cluster can be created. However, this would add to the HAM computational complexity.

Reseting the algorithm may also be necessary for chronic recordings. The clusters size and inter-cluster distance determine whether the clusters should be merged and/or grouped, respectively. For short signals, as the ones that have been used for simulations in this paper, each incoming spike can affect the cluster size and centroid location. However, for chronic recordings, unless weighting is introduced or the stored information is reset, the influence of a new feature vector will be negligible. This is only acceptable if the clusters cen- 
troid location remains practically invariant. This can however be overcome by simply limiting the clusters sizes by scaling down when they reach a certain size, e.g. divide all cluster sizes by 4 whenever any group reaches 1000 spikes.

\section{Conclusion}

This paper has reported a new unsupervised classification method (HAM clustering) for real-time spike sorting. Its key features are that is does not require off-line training (unlike template matching), adaptively determines the number of clusters (unlike $k$-means), achieves high classification accuracy using FSDE and PCA features (comparable to 10 -iterations $k$-means) and has low computational complexity in terms of number of operations (comparable to a single iteration of $k$-means) and memory requirements (requires no historical data). We have demonstrated its operation using multiple datasets, quantified its relatively low complexity and benchmarked its accuracy showing it is comparable to $k$-means. Finally, it has been described how it can be translated to a viable and highly efficient hardware platform.

\section{Acknowledgments}

This work was supported by the UK Engineering and Physical Sciences Research Council (EPSRC Grant refs. EP/I000569/1 and EP/K015060/1). The authors would like to thank Dr. Andrew Jackson (Newcastle University) for providing us with neural recordings used in our realistic test dataset.

\section{Appendix A. Abbreviations in the paper}

Here is an alphabetical list of abbreviations used in this paper.

\section{References}

Abeles M, Goldstein Jr M. Multispike train analysis. IEEE Proceedings 1977;65(5):762-773.

Barsakcioglu D Y, Eftekhar A, Constandinou T G. Design optimisation of front-end neural interfaces for spike sorting systems. In: Circuits and Systems (ISCAS), 2013 IEEE International Symposium on. IEEE; 2013. p. 2501-2504.

Buzsaki G. Large-scale recording of neuronal ensembles. Nature Neuroscience 2004;7(2004):446-451.

Chan H, Wu T, Lee S, Fang S, Chao P, Lin M. Classification of neuronal spikes over the reconstructed phase space. Journal of neuroscience methods 2008;168(1):203-211.

$\begin{array}{ll}\text { ALU } & \text { Arithmetic Logic Unit } \\ \text { ECoG } & \text { Electrocorticography } \\ \text { EEG } & \text { Electroencephalography } \\ \text { FSDE } & \text { First and Second Derivative Extrema } \\ \text { HAM } & \text { Hierarchical Adaptive Means } \\ \text { IA } & \text { Incremental Accuracy } \\ \text { LFPs } & \text { Local Field Potentials } \\ \text { MA } & \text { Moving Accuracy } \\ \text { MCK } & \text { Moving Centroid K-means } \\ \text { MEA } & \text { Microelectrode Array } \\ \text { MIPS } & \text { Million Instructions per Second } \\ \text { NEO } & \text { Non-linear Energy Operator } \\ \text { PCA } & \text { Principle Component Analysis } \\ \text { SPC } & \text { Super Paramagnetic Clustering } \\ \text { SUA } & \text { Single Unit Activity } \\ \text { SNR } & \text { Signal-to-Noise Ratio } \\ \text { ZCF } & \text { Zero Crossing Features }\end{array}$

Chen T-C, Ma T-C, Chen Y-Y, Chen L-G. Low power and high accuracy spike sorting microprocessor with on-line interpolation and re-alignment in 90nm CMOS process. IEEE Engineering in Medicine and Biology Society 2012;:4485-4488.

Dai J, Liu X, Yi Y, Zhang H, Wang J, Zhang S, Zheng X. Experimental study on neuronal spike sorting methods. IEEE Future Generation Communication and Networking Conference 2008;2:230-233.

Eftekhar A, Paraskevopoulou S E, Constandinou T G. Towards a Next Generation Neural Interface: Optimizing Power, Bandwidth and Data Quality. IEEE Biomedical Circuits and Systems Conference 2010;:122-125.

Gibson S, Judy J, Markovic D. Comparison of spike-sorting algorithms for future hardware implementation. IEEE Engineering in Medicine and Biology Society 2008;:5015-5020.

Gibson S, Judy J, Markovic D. Spike sorting: the first step in decoding the brain. IEEE Signal Processing Magazine 2012;29(1):124-143.

Guha S, Meyerson A, Mishra N, Motwani R, O'Callagahan L. Clustering data streams: Theory and practice. IEEE Transactions on Knowledge and Data Engineering 2003;15(3).

Harris K, Henze D, Csicsvari J, Hirase H, Buzsaki G. Accuracy of tetrode spike separation as determined by simultaneous intracellular and extracellular measurements. Journal of Neurophysiology 2000;84(1):401-414.

Jackson A, Fetz E. Compact movable microwire array for long-term chronic unit recording in cerebral cortex of primates. Journal of Neurophysiology 2007;98(5):3109-3118.

Jain A. Data clustering: 50 years beyond k-means. Pattern Recognition Letters 2010;31(8):651-666.

Kamboh A, Mason A. Computationally efficient neural feature extraction for spike sorting in implantable high-density recording systems. IEEE Transactions on Neural Systems and Rehabilitation Engineering 2012;

Karkare V, Gibson S, Markovic D. A 130- $\mu$ W, 64-channel neural spike-sorting DSP chip. IEEE Journal of Solid-State Circuits 2011;46(5):1214-1222.

Karkare V, Gibson S, Markovic D. A $75 \mu \mathrm{W}, 16$-channel neural spikesorting processor with unsupervised clustering. IEEE Journal of Solid-State Circuits 2013;48(9):1-9.

Khalilian M, Mustapha N. Data stream clustering: Challenges and issues. International MultiConference of Engineers and Computer Scientists 2010; 
Kim S, McNames J. Automatic spike detection based on adaptive template matching for extracellular neural recordings. Journal of neuroscience methods 2007;165(2):165-174.

Lebedev M, Nicolelis M. Brain-machine interfaces: past, present and future. Trends in Neuroscience 2006;29(9):536-546.

Letelier J, Weber P. Spike sorting based on discrete wavelet transform coefficients. Journal of neuroscience methods 2000;101(2):93106

Lian J, Garner G, Muessig D, Lang V. A simple method to quantify the morphological similarity between signals. Signal Processing 2010;90(2):684-688.

Mahmud M, Bertoldo A, Girardi S, Maschietto M, Vassanelli S. Sigmate: A matlab-based automated tool for extracellular neuronal signal processing and analysis. Journal of neuroscience methods 2012;

Paraskevopoulou S, Barsakcioglu D, Saberi M, Eftekhar A, Constandinou T. Feature extraction using first and second derivative extrema (fsde) for real-time and hardware-efficient spike sorting. Journal of Neuroscience Methods 2013;215(1):29-37.

Patil P G, Turner D A. The development of brain-machine interface neuroprosthetic devices. Neurotherapeutics 2008;5(1):137-146.

Pedreira C, Martinez J, Ison M, Quiroga R. How many neurons can we see with current spike sorting algorithms? Journal of Neuroscience Methods 2012;211:58-65.

Pouzat C, Mazor O, Laurent G. Using noise signature to optimize spike-sorting and to assess neuronal classification quality. Journal of neuroscience methods 2002;122(1):43-57.

Quiroga R. < http : //www.vis.caltech.edu/rodri/waveclus/waveclushome.htm >

Quiroga R, Nadasdy Z, Ben-Shaul Y. Unsupervised spike detection and sorting with wavelets and superparamagnetic clustering. Neural Computation 2004;16(8):1661-1687.

Rizk M, Bossetti C A, Jochum T A, Callender S H, Nicolelis M A, Turner D A, Wolf P D. A fully implantable 96-channel neural data acquisition system. Journal of neural engineering 2009;6(2):026002

Rutishauser U, Schuman E, Mamelak A. Online detection and sorting of extracellularly recorded action poten- tials in human medial temporal lobe recordings. Journal of Neuroscience Methods 2006;154(1-2):204-224

Saeed M, Kamboh A M. Hardware architecture for on-chip unsupervised online neural spike sorting. IEEE EMBS Conference on Neural Engineering 2013;:1319-1322.

Sarpeshkar R, Wattanapanitch W, Arfin S K, Rapoport B I, Mandal S, Baker M W, Fee M S, Musallam S, Andersen R A. Low-power circuits for brain-machine interfaces. Biomedical Circuits and Systems, IEEE Transactions on 2008;2(3):173-183.

Shoham S, Nagarajan S. Neuroprosthetics: Theory and Practice. World Scientific Publishing, 2004.

Steinbach M, Karypis G, Kumar V. A comparison of document clustering techniques. In: KDD Workshop on Text Mining. 2000.

Stevenson I H, Kording K P. How advances in neural recording affect data analysis. Nature neuroscience 2011;14(2):139-142.

Wild J, Prekopcsak Z, Sieger T, Novak D, Jech R. Performance comparison of extracellular spike sorting algorithms for single-channel recordings. Journal of Neuroscience Methods 2012;203(2012):369-376.

Winterstein F, Bayliss S, Constantinides G A. Fpga-based kmeans clustering using tree-based data structures. In: Field Programmable Logic and Applications (FPL), 2013 23rd International Conference on. IEEE; 2013. p. 1-6.

$\mathrm{Xu}$ R, Wunsch D. Clustering algorithms in biomedical research: A review. IEEE Reviews in Biomedical Engineering 2010;3:120 154.

Yogita, Toshniwal D. Clustering techniques for streaming data - a survey. 3rd IEEE International Advance Computing Conference
2012;(951-956).

Zviagintsev A, Perelman Y, Ginosar R. Low power architectures for spike sorting. In: IEEE Engineering in Medicine and Biology Society. 2005. p. 162-165 